\title{
Establishment and evaluation of a compound fear behavior model of Tourette's syndrome in rats
}

\author{
Qian Chu ${ }^{1 \#}$, Anran Song ${ }^{1 \#}$, Run Zhao ${ }^{1}$, Jianmin Liu ${ }^{2}$, Huishan Shi ${ }^{1}$ Pulin Liu ${ }^{1}$, Chengda Dong ${ }^{1}$, Zhaojun Yan $^{2}$ \\ ${ }^{1}$ College of Traditional Chinese Medicine, Shandong University of Traditional Chinese Medicine, Jinan, China; ${ }^{2}$ Department of Psychosomatic \\ Medicine, Affiliated Hospital of Shandong University of Traditional Chinese Medicine, Jinan, China \\ Contributions: (I) Conception and design: Z Yan; (II) Administrative support: Q Chu; (III) Provision of study materials or patients: A Song; (IV) \\ Collection and assembly of data: R Zhao, C Dong; (V) Data analysis and interpretation: J Liu, P Liu; (VI) Manuscript writing: All authors; (VIII) \\ Final approval of manuscript: All authors. \\ "These authors contributed equally to this work. \\ Correspondence to: Zhaojun Yan. Department of Psychosomatic Medicine, Affiliated Hospital of Shandong University of Traditional Chinese \\ Medicine, Jinan, China. Email: yanzhaojun7790@sina.com.
}

\begin{abstract}
Background: Tourette syndrome (TS) is a common childhood disorder characterized by unwanted movements or vocal sounds called tics. It is often accompanied by other psychobehavioral disorders, including fearful behavior. The establishment and evaluation of rat models of TS and comorbid fear can provide an experimental basis for the treatment of TS and its comorbid fear disorder.

Methods: Sixteen rats were randomly divided into a model group $(n=8)$ and control group $(n=8)$. In the model group, rats were injected intraperitoneally with iminodipropionitrile (IDPN) for 1 week to establish the TS model, which was followed by acoustic and electrical stimulation for 3 weeks to establish the rat models of TS and comorbid fear. The control group received intraperitoneal injection of saline for 1 week, and no further intervention was given in the last 3 weeks. The behavioral changes of the rats were observed and analyzed by the open field test (OFT). Protein kinase A (PKA), cyclic adenosine monophosphate (cAMP), and dopamine (DA) were measured by enzyme-linked immunosorbent assay (ELISA), and tyrosine hydroxylase (TH) and microRNA-134 (miRNA-134) in the brain tissue were detected by using polymerase chain reaction (PCR).
\end{abstract}

Results: One rat in the model group died on the 24th day. Compared with the control group, the model group had significantly higher scores of locomotor activity, stereotyped behavior, and motor behavior, along with prolonged freezing time and significantly lower expression of miRNA-134. The differences in the expressions of PKA, cAMP, DA, and TH in brain tissue were not statistically significant.

Conclusions: The rat models of TS and comorbid fear have similar changes in behaviors and miRNA-134 level to those in clinical settings and therefore can be used as a reliable animal model to study the mechanism of action of TS and comorbid fear.

Keywords: Tourette syndrome (TS); fear; model; microRNA-134 (miRNA-134)

Submitted Jul 09, 2021. Accepted for publication Sep 16, 2021.

doi: $10.21037 / \mathrm{atm}-21-4515$

View this article at: https://dx.doi.org/10.21037/atm-21-4515

\section{Introduction}

Tourette syndrome (TS) is a childhood disorder characterized by unwanted movements or vocal sounds called tics. Studies have pointed out that TS may be related to the disorder of dopaminergic neurotransmitter system or the hyperactivity of dopamine (DA) transporter (1). DA antagonists focus on the basal ganglia to improve TS symptoms (2). The spatial concentration changes of excitatory, inhibitory and regulatory neurochemical ratios in the specific functional system of the basal ganglia may interfere with the 
regulation of the striatum mediated by DA receptors and affect the expression of a variety of motor and non motor characteristics (3). It is often accompanied by a variety of behavioral disorders such as fear, inattention, and obsessivecompulsive disorder (OCD), adding to the complexity and severity of TS itself (4). The study found that parental stress seemed to have little relationship with the frequency and intensity of tic, but more with comorbidity (5). Parents of children with TS need to face many pressures, such as children's social experience, emotional changes, academic problems, family education and financial difficulties caused by diseases (6). Behavioral disorder is the main cause of TS. Clinically, most TS children have fear or timidity and lack of self-confidence. This "fear and fear" may appear before or after convulsive action or sound. However, its etiology and pathophysiology are not clear (7). The lack of feasible animal models is one of the main limiting factors hindering the in-depth study of TS and comorbid fear. Here, we used iminodipropionitrile (IDPN) and acoustic/electrical stimulation to establish rat models of TS and comorbid fear and analyzed and evaluated the behavior changes and the altered expressions of DA, tyrosine hydroxylase (TH), microRNA-134 (miRNA-134), protein kinase A (PKA), and cyclic adenosine monophosphate (cAMP) in brain tissues, aiming to provide an experimental basis for studying the pathology and mechanism of action of TS. We present the following article in accordance with the ARRIVE reporting checklist (available at https://dx.doi.org/10.21037/atm-214515).

\section{Methods}

\section{Experimental animals}

Sixteen male Sprague-Dawley (SD) rats weighing a mean 200 g (SD 2 g) were purchased from Beijing Vital River Laboratory Animal Technology Co., Ltd. [Beijing, China; animal license No. SCXK (Beijing) 2016-0006]. The animals were bred in the Experimental Animal Center of Shangdong University of Traditional Chinese Medicine [license No. SYXK (Lu) 20170022]. The study was approved by the Animal Ethics Committee of Shandong University of Traditional Chinese Medicine Laboratory Animal Center (approval No. SDVTCM20191021002), and the experiment strictly followed the national or institutional guidelines for the care and use of animals. A protocol was prepared before the study without registration.

\section{Main drugs and reagents}

3,3'-IDPN (specification: $25 \mathrm{~g}$ ) was purchased from SigmaAldrich (St. Louis, MO, USA). IDPN was dissolved in normal saline and prepared into a dilution of $100 \mathrm{mg} / \mathrm{mL}$ and stored in the dark. The enzyme-linked immunosorbent assay (ELISA) kits for cAMP, DA, TH, and PKA detection were purchased from J\&L Biological (Shanghai, China).

\section{Main instruments}

The LE116 fear conditioning and startle reflex testing system was purchased from RWD Life Sciences (Shenzhen, China). The H9800 real-time PCR instrument was purchased from Hehui BioTech (Suzhou, China). The absorbance was measured using a spectrophotometer (Techan Group Ltd., Männedorf, Switzerland).

\section{Animal grouping and modeling}

Rats were housed individually at $22 \pm 2{ }^{\circ} \mathrm{C}$ with $50 \% \pm 10 \%$ relative humidity and a 12 -hour light-dark cycle (lights were on from 8 am to $8 \mathrm{pm}$ ). The animals were acclimatized to laboratory conditions for 1 week. These 16 rats were randomly divided into a model group $(\mathrm{n}=8)$ and a control group $(n=8)$ by using the random number table method. TS and comorbid fear models were established in the model group. First, rats were injected intraperitoneally with $300 \mathrm{mg} / \mathrm{kg}$ IDPN once daily for 7 consecutive days to induce TS. After another week of acclimatization, the rats were put into the startle and fear boxes in the fear conditioning and startle reflex testing system. After 5 minutes of adaptation, the rats were exposed to 2 seconds of noise ( 2 beeps), followed by 1 second of electric shock $(2 \mathrm{~mA})$, with an interval of 1 minute. This stimulation was given 30 times a day for 5 consecutive days. On days 6 and 7 , only noise was offered. The experiment was repeated for 3 consecutive weeks, during which the rats were fed a regular diet and water. Rats in the control group received intraperitoneal injections of normal saline for 1 week, and in the remaining 3 weeks they were given regular diet and water without any further intervention.

\section{Specimen sampling}

After 6 weeks, rats in both groups were fasted for 24 hours but with free access to water. After the rats were anesthetized by $2 \%$ of sodium pentobarbital $(50 \mathrm{mg} / \mathrm{kg}$, 
Table 1 The motor behavior and stereotyped behavior scale

\begin{tabular}{lll}
\hline Score & Motor behavior & Stereotyped behavior \\
\hline 0 & Quiet or normal activity & No stereotyped behavior \\
1 & Overexcited & Body rotation (clockwise or counterclockwise) \\
2 & Increased exploratory behavior with & $\begin{array}{l}\text { Excessive up or down movement of the head and neck (an abnormal } \\
\text { movement of the head and neck in the vertical direction to the ground) }\end{array}$ \\
3 & discontinuous sniffing & $\begin{array}{l}\text { Excessive up or down movement of the head and neck plus rotation } \\
\text { Nonstop running }\end{array}$ \\
Nonstop running accompanied by fearful & $\begin{array}{l}\text { Side-to-side head weaving, accompanied by excessive up or down movement } \\
\text { of the head and neck }\end{array}$ \\
\hline
\end{tabular}

intraperitoneally; P3761, 1 g; Sigma-Aldrich). Brain tissues containing the hippocampus, striatum, and other limbic tissues were quickly peeled off on the ice box, weighed, placed in Eppendorf (EP) tubes, and stored at $80^{\circ} \mathrm{C}$ for further tests.

\section{Main measures}

In a quiet environment, the general conditions of the rats, including mental status, activity, and hair color, were observed. Body weight (BW) was measured once weekly.

\section{Open field test (OFT)}

The OFT was performed for comprehensive assessment of tics and fearful behavior. An experimental open box, with black interior and bottom surfaces, was placed in a soundproof environment. The bottom surface of the box was evenly divided into nine compartments, and the temperature inside the box was $20 \pm 0.5^{\circ} \mathrm{C}$. A camera was placed directly above the central area, and its field of view was able to cover the entire interior of the open box. The rats were moved into the test room 1 hour before the start of the experiment to adapt to the environment and then placed in a fixed position in the central zone of the experimental open box, with their heads fixed to a direction during each placement. The total distance of movement, residence time in the central area, the number of times of entering the central area/standing upright/grooming/defecating/urinating, and the 5-minute behaviors were recorded. The distance of movement and residence time in the central area as well as the percentage of horizontal movement in the central area and the percentage of the time spent in the central area were calculated.

\section{Testing for motor behavior and stereotyped behavior}

The motor behavior and stereotyped behavior of the rats were scored using the method described by Steeves et al. (8) (Table 1). One hour after the final medication, rats were acclimatized to the test chamber for 5 minutes, which was followed by double-blinded observation for 1 hour. The results were recorded every 5 minutes, and the means of their total scores were calculated.

\section{Testing for fearful behavior}

Fear was measured by observation of "freezing" behavior (i.e., voluntary immobility of the mouse in seconds). The freezing behavior was tested 48 hours after the final fear modeling session. The main indicators were the following: (I) freezing, including maintaining a fixed body position without moving; (II) small range of exploratory movements, including exploratory movements of the trunk and forelimbs only; and (III) activities, including movement of the hind limbs, hair grooming, and standing. After each measurement of a rat, the excretions in the box were cleaned with $75 \%$ alcohol to eliminate their impact on the activities of the next rat.

\section{Determination of PKA, cAMP, and DA expressions in brain tissues}

DA, PKA, and cAMP protein expressions in brain tissue were detected by ELISA. Blank wells, standard sample wells, and sample wells were set up for testing, and $50 \mu \mathrm{L}$ of various concentrations of standards were added to each well. Additionally, $100 \mu \mathrm{L}$ of ELISA reagent was added to each standard sample well and sample well. The wells were incubated for 60 minutes at $37^{\circ} \mathrm{C}$ after the plate was closed 
with the closure plate membrane. Next, the liquid was discarded, the well was dried, and then liquid was added to each well. After these steps were repeated 5 times, the plates were dried, and color developer was added to each well. The solution was fully centrifuged and placed in the dark for color development at $37{ }^{\circ} \mathrm{C}$ for 15 minutes. After this, $50 \mu \mathrm{L}$ of termination solution was added to each well to terminate the reaction. Optical density (OD) was measured for each well.

\section{Determination of miRNA-134 and TH expressions in brain tissues}

Real-time PCR for the detection of TH and miRNA-134 in brain tissues.

\section{Total RNA extraction}

After the harvested brain tissue samples were ground in liquid nitrogen, $1 \mathrm{~mL} / 30-50 \mathrm{mg}$ of TRIzol Reagent was added to lyse the cells. Samples were allowed to stand for 5 minutes at room temperature to ensure complete dissociation of nucleoprotein complexes. The mixture was centrifuged at $4{ }^{\circ} \mathrm{C}$ at $12,000 \mathrm{rpm}$ for 5 minutes. The supernatant was then harvested, mixed with $200 \mu \mathrm{L}$ of chloroform, and left to stand for 2 minutes. After centrifugation for 10 minutes, the upper aqueous phase was taken and anhydrous ethanol was added into the adsorption column.

The obtained liquid was added to the adsorption column. The sample was centrifuged at 12,000 rpm for 20 seconds. The flow-through was discarded, and the adsorption column was placed back into the same tube. After this, $700 \mu \mathrm{L}$ of RW1 wash buffer was added to the adsorption column. After centrifugation at 12,000 rpm for 10 minutes, the flow-through was discarded, and adsorption column was placed back into the same tube. After this, $500 \mu \mathrm{L}$ of RW2 wash buffer was added to the adsorption column, and the sample was centrifuged at 12,000 rpm for 20 seconds. The above step was repeated twice, and then the adsorption column was air dried.

\section{RNA reverse transcription to generate complement} DNA (cDNA)

For RNA reverse transcription, $1 \mu \mathrm{L}$ of oligo (dT) primers $(0.5 \mu \mathrm{g} / \mu \mathrm{L}), 2.0 \mu \mathrm{g}$ of total RNA, and RNase-Free $\mathrm{H} 2 \mathrm{O}$ were mixed well and centrifuged. The sample was incubated for 10 minutes at $70{ }^{\circ} \mathrm{C}$ in a water bath, which was immediately followed by an ice bath of the anneal oligo (dT) primers and template. The reverse transcriptase (RT) was inactivated with a water bath, and the obtained RT product- $\mathrm{CDNA}$ - was stored at $-80{ }^{\circ} \mathrm{C}$ for subsequent tests (9). Real-time polymerase chain reaction (PCR) proceeded as follows: SYBR premix ex taq $(10 \mu \mathrm{L})$, upstream primer $(0.5 \mu \mathrm{L})$, downstream primer $(0.5 \mu \mathrm{L})$, cDNA $(1 \mu \mathrm{L})$, and $\mathrm{RNase}-F r e e \mathrm{H}_{2} \mathrm{O}(8 \mu \mathrm{L})$ were well mixed before being loaded into a PCR instrument for amplification, and thus the concentrations of $\mathrm{TH}$ and miRNA-134 were determined. The upstream primer of miRNA-134 was GATTTGGCCGTATCGGAC, and the downstream primer was GAAGACGCCAGTAGACTC; the upstream primer of TH was GCGACAGAGTCTCATCGAGG, and the downstream primer was AGCATTCCCATCCCTCTCCT.

\section{Statistical analysis}

Statistical analysis was performed by using the SPSS 25.0 software package (IBM Corporation, Armonk, NY, USA). The comparisons between two groups that conformed to normal distribution were conducted by independent samples $t$-test, while the skewed data were analyzed by rank-sum test. Comparisons of two groups at different time points were conducted by the repeated-measures analysis of variance (ANOVA). A P value of $<0.05$ was considered significantly different.

\section{Results}

\section{General status of rats in the two groups}

There was no significant difference in the general status of the rats in the two groups before and 7 days after modeling. Compared with the control group, the model group had poor mental status, more yellow or dark hair, decreased BW, and reduced activities 14, 21, and 28 days after modeling; one rat in the model group died on the 24th day.

\section{Behavioral comparisons of the two groups in the OFT}

The OFT showed that the percentage of horizontal movement in the central area and the percentage of time spent in the central area were significantly different between the model group and control group $(\mathrm{P}<0.05 ;$ Table 2).

\section{Motor behavior and stereotyped behavior}

Motor behavior and stereotyped behavior significantly increased after modeling in the model group compared with 
Table 2 Results of the OFT in the two groups

\begin{tabular}{|c|c|c|c|c|}
\hline Time & Number of rats & Group & $\begin{array}{l}\text { Percentage of horizontal movement } \\
\text { in the central area }\end{array}$ & $\begin{array}{l}\text { Percentage of time spent in the } \\
\text { central area }\end{array}$ \\
\hline \multirow[t]{2}{*}{ Week 1} & 8 & Control group & $0.54 \pm 1.31$ & $0.23 \pm 0.62$ \\
\hline & 7 & Model group & $8.36 \pm 7.54$ & $3.31 \pm 3.66$ \\
\hline Week 2 & 8 & Control group & $4.19 \pm 6.44$ & $0.51 \pm 0.53$ \\
\hline Week 3 & 7 & Model group & $2.24 \pm 2.51$ & $4.55 \pm 2.51$ \\
\hline \multirow[t]{2}{*}{ Week 4} & 8 & Control group & $3.77 \pm 2.89$ & $2.81 \pm 3.15$ \\
\hline & 7 & Model group & $9.02 \pm 5.39$ & $10.09 \pm 15.61$ \\
\hline Week 6 & 7 & Model group & $8.83 \pm 11.63$ & $4.66 \pm 4.36$ \\
\hline
\end{tabular}

OFT, open field test.

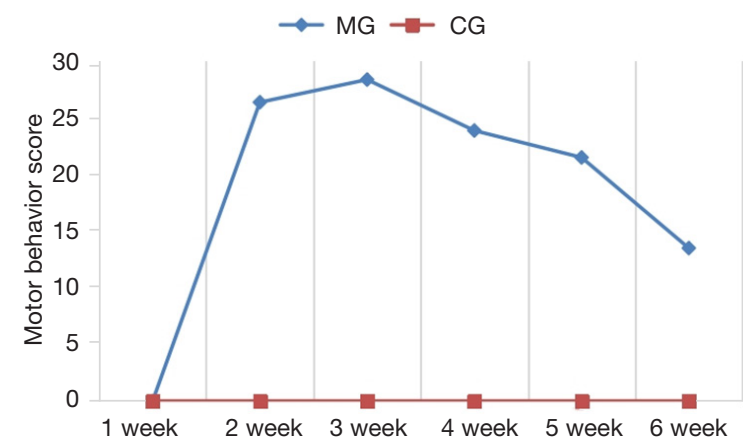

Figure 1 Comparison of motor behavior between the two groups. MG, model group; CG, control group.

the control group $(\mathrm{P}<0.05$; Figures 1,2$)$.

\section{Fearful behaviors}

Compared with the control group, the model group showed prolonged freeing after modeling $(\mathrm{P}<0.05$; Figure 3).

\section{Expressions of miRNA-134, PKA, cAMP, TH, and DA in brain tissues}

There was no statistically significant difference in the expression of PKA, cAMP, TH, or DA in the brain tissues between the model group and control group (all

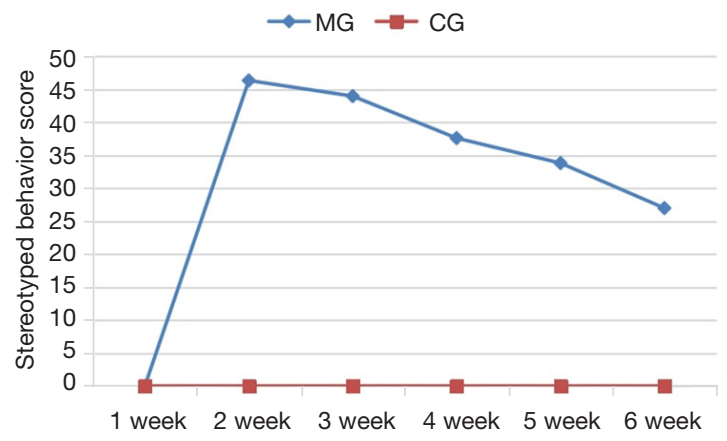

Figure 2 Comparison of stereotyped behavior between the two groups. MG, model group; CG, control group.

$\mathrm{P}$ values $>0.05)$. However, miRNA-134 expression was significantly lower in the model group than in the control group $(\mathrm{P}<0.05$; Figures 4-8; Table 3).

\section{Discussion}

TS is a common psychobehavioral disorder in children, with genetic factors, neurotransmitter abnormalities, and environmental factors being the possible causes. With the increasing incidence of TS, management of TS and its comorbidities has become an urgent issue. The lifetime prevalence of any psychiatric comorbidity among individuals with TS has been found to be $85.7 \%$, with 


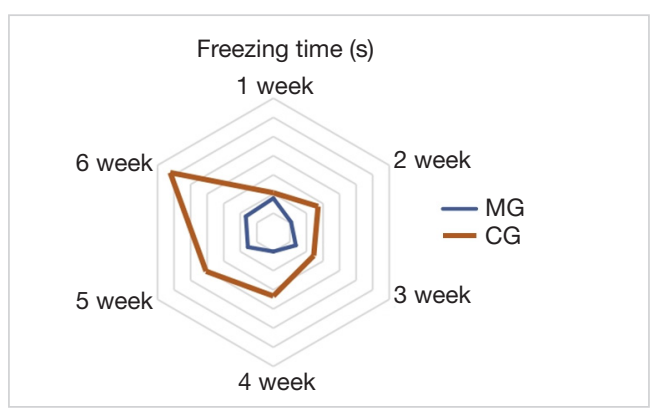

Figure 3 Comparison of freezing time between the two groups. MG, model group; CG, control group.

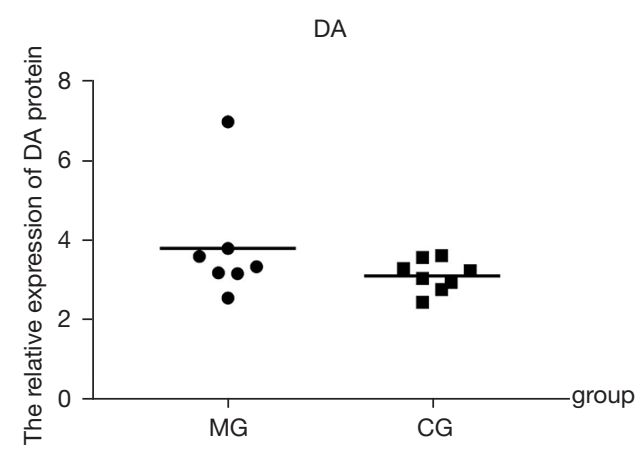

Figure 4 DA expression in two groups. DA, dopamine; MG, model group; CG, control group.

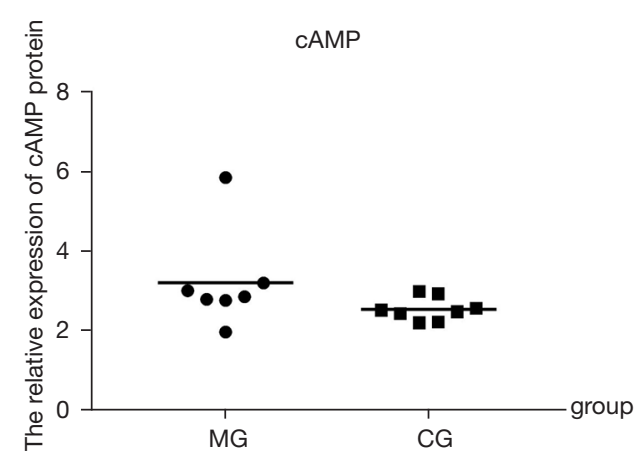

Figure 5 cAMP expression in the two groups. cAMP, cyclic adenosine monophosphate; MG, model group; CG, control group.

$29.7 \%$ of children having comorbidities including phobias and posttraumatic stress disorder (PTSD) and OCD and attention-deficit/hyperactivity disorder (ADHD). The high complication rate indicates that there is a common neurobiological basis, which may be related to more serious injury and more operational difficulties. In recent decades, people have studied its potential neurobiological mechanism,

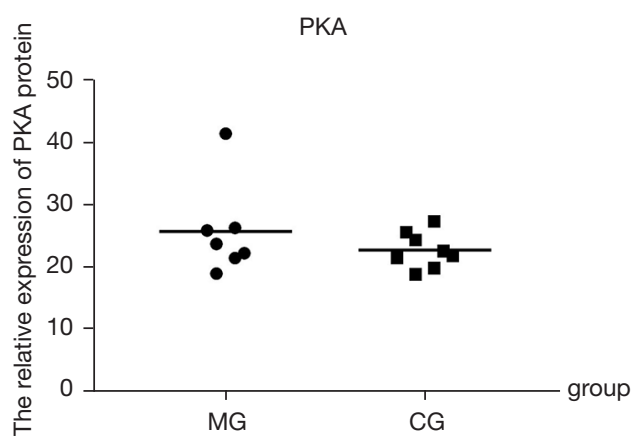

Figure 6 PKA expression in the two groups. PKA, protein kinase A; MG, model group; CG, control group.

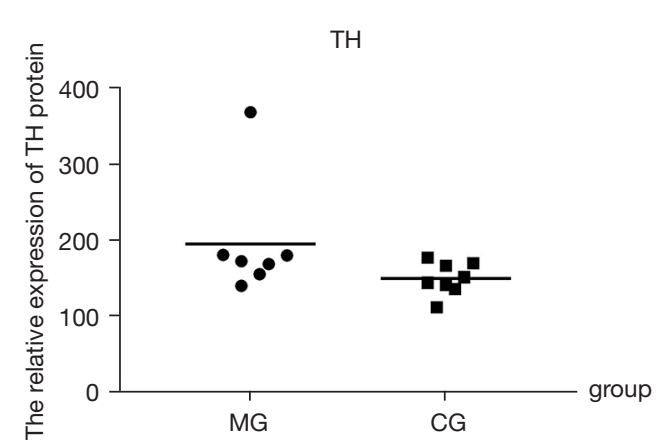

Figure $7 \mathrm{TH}$ expression in the two groups. TH, tyrosine hydroxylase; MG, model group; CG, control group.

but no exact answer has been obtained (10). Suitable animal models can provide a basis for understanding the potential mechanisms of action of TS combined with fear-related behaviors and for developing effective treatments.

At present, there are a number of studies on TS or fear alone, and methods for constructing corresponding animal models have been well documented. Notably, IDPNinduced TS models have been widely used in scientific research. As a central neurotoxin, IDPN can disrupt the DA system in the extrapyramidal system and maintain DA at a low concentration for a long period of time; this causes the model rats to develop DA hypersensitivity during their growth and development, leading to the appearance of tics (11). The fear conditioning models based on Pavlovian fear conditioning are the most widely recognized and applied animal models of fear memory (12); during their creation, a fear response is induced by establishing multiple connections between electrical shock and sound. Rat models of TS and comorbid fear were established in our study. One week after intraperitoneal injection of IDPN, the rats 
The relative RNA expression of miRNA134

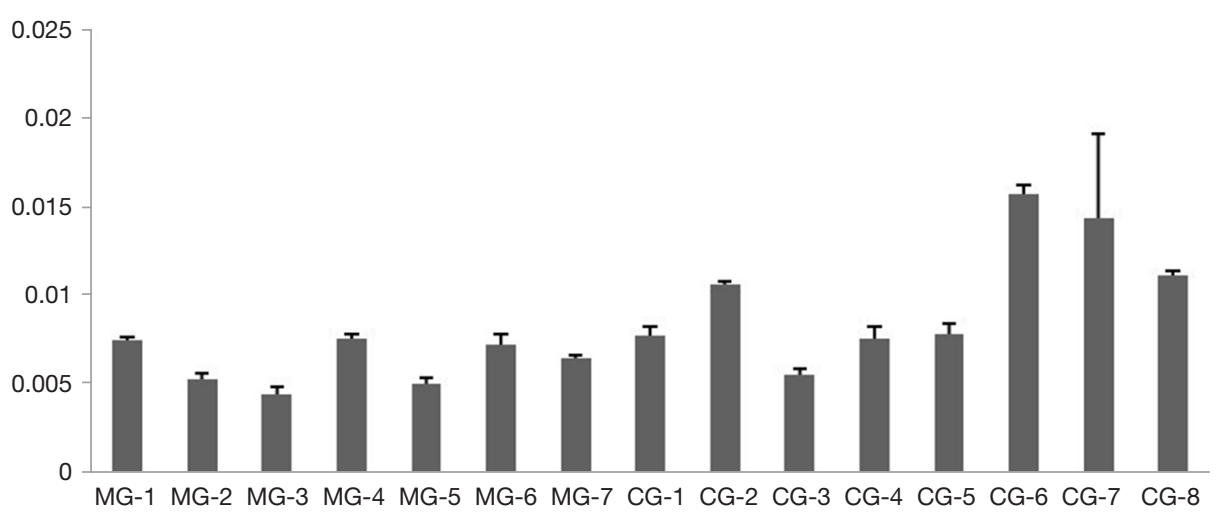

Figure 8 miRNA-134 expression in the two groups. miRNA-134, microRNA-134; MG, model group; CG, control group.

Table 3 Comparisons of expressions in brain tissues between the two groups

\begin{tabular}{lccccc}
\hline Group & DA & cAMP & PKA & TH & miRNA-134 \\
\hline Control group & $378.77 \pm 145.66$ & $3.18 \pm 1.24$ & $25.77 \pm 7.42$ & $195.80 \pm 77.74$ & $0.0062 \pm 0.0013$ \\
Model group & $309.96 \pm 39.85$ & $2.51 \pm 0.29$ & $22.77 \pm 2.89$ & $150.56 \pm 21.31$ & $0.0100 \pm 0.0036$ \\
$\mathrm{t}$ & 1.289 & 1.490 & 1.060 & 1.587 & -2.691 \\
$\mathrm{P}$ & 0.220 & 0.160 & 0.308 & 0.136 & 0.019 \\
\hline
\end{tabular}

DA, dopamine; cAMP, cyclic adenosine monophosphate; PKA, protein kinase A; TH, tyrosine hydroxylase; miRNA-134, microRNA-134.

in the model group exerted reduced locomotor activity and increased motor behavior and stereotyped behaviors compared with the control group, indicating successful TS modeling. Subsequently, the model group was stimulated by electric shock and noise in a fear conditioning and startle reflex testing system. The freezing time of rats in the model group was prolonged. During the modeling, the rats showed obvious tic and fear-like behaviors such as nodding, twisting body, vocalizing, repeated grooming, and dullness, indicating the successful creation of TS and comorbid fear models. Histidine decarboxylase (HDC) gene mutation is a rare genetic cause of TS. HDC knockout mice are an important animal experimental model to study the phenomenology and pathophysiology of TS disease. Both experimental models used tone as fear conditioned reflex to enhance animal stereotype, and also studied the regulatory effect of Da. However, the rat model constructed in this paper uses not only tone as fear conditioned reflex, but also electric shock as shock reflex, which increases the success of the animal experimental model $(13,14)$.

The DA hypothesis can largely explain the mechanism of action of TS. It has been suggested that DA receptor hypersensitivity, DA transporter hyperactivity, presynaptic dysfunction of DA neurons, and excessive dopaminergic innervation can lead to altered expression and learning and abnormal twitching behavior (15). TH is the ratelimiting enzyme for DA biosynthesis. Variations in the $\mathrm{TH}$ gene may play a role in the pathogenesis of TS (16), and downregulation of $\mathrm{TH}$ gene levels can lead to the decrease of DA receptor expression (17). PKA can activate the cAMP2-responsive element-binding protein (CREB) and induce gene transcription upon binding to a response factor in the promoter region of the TH gene, thereby regulating TH activity (18). By being involved in both short- and long-term regulation of TH, PKA causes TH to undergo phosphorylation, increases the catalytic rate of the enzyme, and enhances the synthesis of DA. In contrast, disturbances in any of the component processes (including synthesis, storage, release, and degradation) of the neurotransmitter life cycle or any functional abnormality of transmitter receptors can lead to corresponding alterations (e.g., fearful behavior) in neuropsychiatric functions (19). In the present study, there was no significant difference in the expression of DA, TH, PKA, or cAMP in the model group compared 
with the control group. It is speculated that IDPN induction for establishing a TS model leads to a decrease in DA content, while acoustic and electrical stimulation for inducing a fear model causes a stress-specific increase in DA expression; these two processes eventually offset each other, leading to no significant change in DA content. As there was no change in DA expression, TH might not have affected DA. Furthermore, PKA and cAMP might not have affected the phosphorylation of TH. As a result, the expressions of DA, TH, PKA, and cAMP did not change significantly in the model group.

miRNAs are involved in the development of axons, dendrites, and synapses, and in neuronal proliferation, thereby playing important roles in learning, memory, emotions, and sensory functions $(20,21)$. Some scholars have studied, TS patients have mutations in the 3 ' untranslated region (3'UTR) of SLITRK1 gene at mir-189 binding site (22). And miR-429 expression was reduced in serum of TS patients, and miR-429 may be immediately useful as sensitive molecular biomarker to support TS diagnosis (23). In a fear study (24), the fear memory was enhanced by downregulating miRNA in the hippocampal region of mice after classic contextual fear conditioning (CFC) training. miR-134 has been reported to be a brain-specific miRNA. As a member of the transcription factor protein family, CREB is localized within the nuclei of all cells in the brain and participates in multiple signaling pathways. The biological activity of CREB protein is regulated by phosphorylation, which enhances the transcriptional activity of CREB. miR-134 regulates CREB protein expression in a posttranscriptional manner. miR-134 normally functions to limit the expression of CREB protein, and overexpression of miR-134 inhibits the expression of CREB $(25,26)$. In our current study, after the creation of TS and comorbid fear models, the miRNA-134 expression was lower in the model group than in the control group, suggesting that downregulated miRNA-134 expression is involved in or interferes with the development of tics and fear memory.

In summary, the interaction between TS and fear contributes to the development of these two conditions. Our current experiment provides a new insight for future studies on TS and comorbid fear. However, our study was limited by its small sample size and the fact that only the brain tissues after last treatment were used for comparisons. In our future studies, the sample sizes will be enlarged, and the intensity of fear stimuli will be stratified to explore the effects of fear stimuli with different intensities on modeling.

\section{Acknowledgments}

Funding: Grants supported this study from the National Natural Science Foundation of China (No. 81774249).

\section{Footnote}

Reporting Checklist: The authors have completed the ARRIVE reporting checklist. Available at https://dx.doi. org/10.21037/atm-21-4515

Data Sharing Statement: Available at https://dx.doi. org/10.21037/atm-21-4515

Conflicts of Interest: All authors have completed the ICMJE uniform disclosure form (available at https://dx.doi. org/10.21037/atm-21-4515). Dr. ZY reports grants support from the National Natural Science Foundation of China (No. 81774249). The other authors have no conflicts of interest to declare.

Ethical Statement: The authors are accountable for all aspects of the work in ensuring that questions related to the accuracy or integrity of any part of the work are appropriately investigated and resolved. The study was approved by the Animal Ethics Committee of Shandong University of Traditional Chinese Medicine Laboratory Animal Center (approval No. SDVTCM20191021002), and the experiment strictly followed the national or institutional guidelines for the care and use of animals.

Open Access Statement: This is an Open Access article distributed in accordance with the Creative Commons Attribution-NonCommercial-NoDerivs 4.0 International License (CC BY-NC-ND 4.0), which permits the noncommercial replication and distribution of the article with the strict proviso that no changes or edits are made and the original work is properly cited (including links to both the formal publication through the relevant DOI and the license). See: https://creativecommons.org/licenses/by-nc-nd/4.0/.

\section{References}

1. Buse J, Schoenefeld K, Münchau A, et al. Neuromodulation in Tourette syndrome: dopamine and beyond. Neurosci Biobehav Rev 2013;37:1069-84.

2. Albin RL. Tourette Syndrome as a Disorder of the Social Decision Making Network. Front Psychiatry 2019;10:742. 
3. Kanaan AS, Gerasch S, García-García I, et al. Pathological glutamatergic neurotransmission in Gilles de la Tourette syndrome. Brain 2017;140:218-34.

4. Wood BL, Klebba K, Gbadebo O, et al. Pilot study of effect of emotional stimuli on tic severity in children with Tourette's syndrome. Mov Disord 2003;18:1392-5.

5. Stewart SB, Greene DJ, Lessov-Schlaggar CN, et al. Clinical correlates of parenting stress in children with Tourette syndrome and in typically developing children. J Pediatr 2015;166:1297-302.e3.

6. Ludlow AK, Brown R, Schulz J. A qualitative exploration of the daily experiences and challenges faced by parents and caregivers of children with Tourette's syndrome. J Health Psychol 2018;23:1790-9.

7. Hedderly T. Fear of fear, severe tics and deep brain stimulation in childhood Tourette Syndrome: The long and winding road. Eur J Paediatr Neurol 2017;21:16-17.

8. Steeves TD, Fox SH. Neurobiological basis of serotonindopamine antagonists in the treatment of Gilles de la Tourette syndrome. Prog Brain Res 2008;172:495-513.

9. Hirschtritt ME, Lee PC, Pauls DL, et al. Lifetime prevalence, age of risk, and genetic relationships of comorbid psychiatric disorders in Tourette syndrome. JAMA Psychiatry 2015;72:325-33.

10. Wanderer S, Roessner V, Strobel A, et al. WISCIV performance of children with chronic tic disorder, obsessive-compulsive disorder and attention-deficit/ hyperactivity disorder: results from a German clinical study. Child Adolesc Psychiatry Ment Health 2021;15:44.

11. Wang Y, Li A. Regulatory effects of Ningdong granule on dopaminergic and serotonergic neurotransmission in a rat model of Tourette syndrome assessed by PET. Mol Med Rep 2019;20:191-7.

12. Nasser HM, McNally GP. Neural correlates of appetitiveaversive interactions in Pavlovian fear conditioning. Learn Mem 2013;20:220-8.

13. Xu M, Li L, Ohtsu H, et al. Histidine decarboxylase knockout mice, a genetic model of Tourette syndrome, show repetitive grooming after induced fear. Neurosci Lett 2015;595:50-3.

14. Baldan LC, Williams KA, Gallezot JD, et al. Histidine decarboxylase deficiency causes tourette syndrome: parallel findings in humans and mice. Neuron 2014;81:77-90.

15. Maia TV, Conceição VA. Dopaminergic disturbances in Tourette syndrome: an integrative account. Biol Psychiatry 2018;84:332-44.

16. Comings DE, Gade R, Muhleman D, et al. No association of a tyrosine hydroxylase gene tetranucleotide repeat polymorphism in autism, Tourette syndrome, or ADHD. Biol Psychiatry 1995;37:484-6.

17. Lin L, Yu L, Xiang H, et al. Effects of acupuncture on behavioral stereotypies and brain dopamine system in mice as a model of Tourette syndrome. Front Behav Neurosci 2019;13:239.

18. Zhen JL, Chang YN, Qu ZZ, et al. Luteolin rescues pentylenetetrazole-induced cognitive impairment in epileptic rats by reducing oxidative stress and activating PKA/CREB/BDNF signaling. Epilepsy Behav 2016;57:177-84.

19. Jin Z, Yan ZJ, Li YQ, et al. Effect of "Qiangzhizufang" on the rat model of Tourette syndrome combined with fear. Chinese Journal of Comparative Medicine 2016;26:71-6, 82.

20. Bredy TW, Lin Q, Wei W, et al. MicroRNA regulation of neural plasticity and memory. Neurobiol Learn Mem 2011;96:89-94.

21. Konopka W, Schütz G, Kaczmarek L. The microRNA contribution to learning and memory. Neuroscientist 2011;17:468-74.

22. Melo-Felippe FB, Fontenelle LF, Kohlrausch FB. Gene variations in PBX1, LMX1A and SLITRK1 are associated with obsessive-compulsive disorder and its clinical features. J Clin Neurosci 2019;61:180-5.

23. Rizzo R, Ragusa M, Barbagallo C, et al. Circulating miRNAs profiles in Tourette syndrome: molecular data and clinical implications. Mol Brain 2015;8:44.

24. Zhao C, Zhou B, Cao J, et al. miR-187-3p participates in contextual fear memory formation through modulating SATB2 expression in the hippocampus. Neuroreport 2020;31:909-17.

25. Campbell DS, Holt CE. Chemotropic responses of retinal growth cones mediated by rapid local protein synthesis and degradation. Neuron 2001;32:1013-26.

26. Gao J, Wang WY, Mao YW, et al. A novel pathway regulates memory and plasticity via SIRT1 and miR-134. Nature 2010;466:1105-9.

(English Language Editor: J. Gray)

Cite this article as: Chu Q, Song A, Zhao R, Liu J, Shi H, Liu P, Dong C, Yan Z. Establishment and evaluation of a compound fear behavior model of Tourette's syndrome in rats. Ann Transl Med 2021;9(18):1469. doi: 10.21037/atm-21-4515 